DOI: 10.17516/1997-1397-2021-14-3-344-350

УДК 512.54

\title{
Sharply 3-transitive Groups with Finite Element
}

\author{
Evgeny B. Durakov* \\ Siberian Federal University
}

Krasnoyarsk, Russian Federation

Received 31.12.2020, received in revised form 16.01.2021, accepted 20.02.2021

Abstract. In this paper we study sharply 3-transitive groups. The local finiteness of sharply triply transitive permutation groups of characteristic $p>3$ containing a finite element of order $p$ is proved.

Keywords: group, sharply k-transitive group, sharply 3-transitive group, locally finite group, neardomain, near-field.

Citation: E.B. Durakov, Sharply 3-transitive Groups with Finite Element, J. Sib. Fed. Univ. Math. Phys., 2021, 14(3), 344-350. DOI: 10.17516/1997-1397-2021-14-3-344-350.

\section{Introduction}

We recall that the group $G$ of permutations of the set $F(|F| \geqslant k)$ is called exactly $k$-transitive on $F$ if for any two ordered sets $\left(\alpha_{1}, \ldots, \alpha_{k}\right)$ and $\left(\beta_{1}, \ldots, \beta_{k}\right)$ elements from $F$ such that $\alpha_{i} \neq \alpha_{j}$ and $\beta_{i} \neq \beta_{j}$ for $i \neq j$, there is exactly one element of the group $G$ taking $\alpha_{i}$ to $\beta_{i}(i=1, \ldots, k)$.

In $1872, \mathrm{~K}$. Jordan described the class of finite sharply $k$-transitive groups for $k \geqslant 4([1$, page 215]).

In infinite groups J. Tits and M. Hall established that for $k \geqslant 4$ infinite sharply $k$-transitive groups do not exist ( [1, page 215], [2, page 86-87]).

Unlike the cases $k \geqslant 4$, the sets of finite exactly 2- and 3-transitive groups are countable, and the locally finite sets are continuous.

Sharply 2- and 3-transitive groups are closely related algebraic structures such as near-fields, near-domains, $K T$-fields (Kerby-Tits fields), etc. (see [1, Ch. V], [2, chap. 20]).

Finite exactly 2 - and 3-transitive groups and near-fields were classified by G. Zassenhaus [1, ch. IV and Theorem V.5.2]. Complete description of locally finite sharply 3-transitive groups in 1967 got O. Kegel [3].

The study of the class of infinite exactly 2- and 3-transitive groups is actively continued at the present time. In 2000 V. D. Mazurov in [4] fully described exactly 3 - transitive groups with abelian stabilizers of two points. In 2011, T. Grundhöfer and E. Jabara proved the local finiteness of the binary finite sharply doubly transitive groups [5]. In 2013, in the paper [6], A. I. Sozutov established a similar fact for the periodic groups of Shunkov.

In the paper [7], in the class of sharply triply transitive groups, the local finiteness of permutation groups with a periodic stabilizer of two points was proved and, as a consequence, the local finiteness of the periodic sharply 3-transitive groups.

In the papers $[8,9]$, examples of sharply doubly transitive groups of characteristic 2 that do not contain regular abelian normal subgroups are constructed, and in [10], there are similar examples of sharply 3-transitive groups. These examples show that there are near-domains of characteristic 2 that are not near-fields and $K T$-fields, $(F, \sigma)$, in which near-domains $(F,+, \cdot)$ are not near-fields. This provides a basis for studying these structures with additional restrictions.

*durakov@mail.ru

(c) Siberian Federal University. All rights reserved 
Recall that a nonidentity element $k$ of a group $G$ is called finite in $G$ if for any $g \in G$ the subgroup $\left\langle k, k^{g}\right\rangle$ is finite.

Let $G$ be sharply 3-transitive on $X, J$ the set of involutions in $G, J^{2}=\{k v \mid k, v \in J\}$. The characteristic $G(\operatorname{Char}(G))$ is defined as follows [1]:

1. $\operatorname{Char}(G)=2$, if elements from $J$ do not fix points from $X$;

2. $\operatorname{Char}(G)=0$ if each $g \in J^{2} \backslash\{1\}$ is of infinite order;

3. $C h a r(G)=p$, where $p$ is odd prime, if the order of each $g \in J^{2} \backslash\{1\}$ is $p$.

In continuation of the research started in [7] and [11], in this work a special case of Theorem 6 announced in [12] is proved:

Theorem 1. A sharply triple transitive permutation group of characteristic $p>3$, containing a finite element of order $p$, is locally finite.

\section{Proof of the theorem}

Let $G$ be an infinite sharply triply transitive permutation group of the set $X=F \cup\{\infty\}$. By $B$ we denote the stabilizer $G_{\alpha}$ of the point $\alpha \in X$ and through $H$ - stabilizer $G_{\alpha \beta}=G_{\alpha} \cap G_{\beta}$ of two points $\alpha=\infty \in X, \beta \in F$. Let also $J$ be the set of involutions of the group $G$, and $J_{m}$ be the set involutions stabilizing exactly $m$ points, $m=0,1,2$. Let us also formulate the well-known properties of involutions from groups $G=T_{3}(F, v)$ and $B=T_{2}(F)$ (see, for example, [1, Ch. V]) with comments.

Lemma 1. The following statements are true:

1. The group $B=G_{\infty}$ is regular on the set $F$ an elementary abelian p-subgroup of $U$ and $B=U \lambda H-$ Frobenius group.

2. $U$ - Sylow $p$-subgroup of the group $G, B=N_{G}(U), U^{\#}=a^{H}, C_{G}(u)=U$ for any element $u \in U^{\#}$ and $U \cap U^{x}=1$ for any element $x \in G \backslash B$.

3. $H=G_{\infty} \cap G_{\alpha}, H$ contains the only involution $z, z \in J_{2}, C_{G}(z)=N_{G}(H)$.

4. Each subgroup of order qr in $H$, where $q, r$ not necessarily different primes, cyclic, and $H \cap H^{x}=1$ for any element $x \in G \backslash N_{G}(H)$.

5. $N=N_{G}(H)=H \lambda\langle v\rangle$, where $v$ is an involution from $J_{2}, C_{H}(v)=\langle z\rangle$.

6. If $N \cap N^{x} \neq 1$ for $x \in G \backslash N$, then $N \cap N^{x}=\langle t\rangle$, where $t=t(x)$ is an involution.

7. $G=B \cup B v U$ and $B \cap B^{x}=H^{b}$ for any $x \in G$ setminus $B$ and a suitable $b=b(x) \in B$.

Proof. 1. The statement follows from [6, Theorem 2].

2. The statement easily follows from the exact 3-transitivity of $G$ (see also [7, Lemma 1], [13], item 1 of the lemma and finiteness of elements from $U$. Non-trivial element from $U \cap U^{x}$ must stabilize two points, which is impossible in view of item 1.

3. The statement is well known $[1,6,14]$.

4. The statement follows from Burnside's theorem [15, Theorem 1.2], 3-transitivity of $G$ and equality $B \cap B^{x}=G_{\infty} \cap G_{\infty^{x}}$.

5. This statement and statement 6 are obvious. 
7. Follows from 2- (and even 3-) transitivity and items 1,5 of the lemma.

The lemma is proved.

The groups $H$ and $N=C_{G}(z)$ will also be denoted by $H_{z}$ and $N_{z}$, and for $k=z^{g}$ by $H_{k}$ and $N_{k}$ we will denote subgroups $H^{g}$ and $N^{g}$.

Lemma 2. The following statements are true:

1. Either $J=J_{2}$, or $J=J_{0} \cup J_{2}$, while $J_{2}=v^{G}$.

2. For each involution $j$ the set $v N \cap j^{G}$ is infinite.

3. For each involution $j \in J$ the set $J_{2} \cap C_{G}(j)$ is infinite.

4. Every Sylow 2-subgroup in $H$ is (locally) cyclic, or (locally) quaternionic; are they conjugate, isomorphic, we do not know yet.

5. Every Sylow 2-subgroup of $T$ from $N$ whose order is greater than 4, is a Sylow 2-subgroup of $G$.

6. If a Sylow 2-subgroup $T$ of $N$ is a proper subgroup of a Sylow 2-subgroup $R$ of $G$, then $R$ is a (locally) dihedral group.

7. $G$ contains no elementary abelian subgroups of order 8 , containing an involution from $J_{2}$. The rank of Sylow 2-subgroups in $N$ is 2. The rank of any Sylow 2-subgroup of $G$ containing an involution from $J_{2}$, is equal to 2 .

Proof. 1. The inequalities $0 \leqslant m \leqslant 2$ follow from the sharply 3 -transitivity of the group $G$. Lemma 1 implies that the partitions $J=J_{1} \cup J_{2}$ and $J=J_{0} \cup J_{1} \cup J_{2}$ are impossible, and it is obvious that the sets $J_{1}$ and $J_{2}$ are conjugacy classes. Since Char $G=p>2$, then either $J=J_{2}$ or $J=J_{0} \cup J_{2}$.

2. In each such class $j^{G}$ there is an involution $k$ permuting the points $\alpha$ and $\beta$. Further, we apply Ditzmann's lemma [16, Lemma 2.3].

3. The involution $j$ is contained in the subgroup $N_{\gamma \delta}$, if the permutation $j$ contains a cycle $(\gamma \delta)$.

4. Follows from Shunkov's theorem [16, Theorem 2.15].

5. The subgroup $\langle z\rangle$ is characteristic in $T$ and $x \in N_{G}(T)$ implies $x \in N=C_{G}(z)$.

6. Follows from the fact that $C_{R}(z)=T$. In particular, potentially $R$ can be an infinite locally dihedral group.

7. If $E_{8} \leqslant N$, then $H \cap E_{8}=E_{4}$, which contradicts the uniqueness of the involution $z$. Further we use item 6 of the lemma. The lemma is proved.

Lemma 3. The set of all 2-elements of the group $H$ invertible involution $v$, is a (locally) cyclic 2-subgroup of $S$. If $x \in H \backslash S$ and $x^{2} \in S$, then the order of the element $x^{-1} v x v$ is infinite.

Proof. The assertions of the lemma are proved in [13, Lemmas 5, 6]

By the conditions of the theorem, all subgroups $L_{x}=\left\langle a, a^{x}\right\rangle$ in $G$ are finite, and for $x \in J$, the subgroups $K_{x}$ are also finite. Let's find out their structure. Let's start with the subgroups $L=\left\langle a, a^{v}\right\rangle, K=\langle a, v\rangle$.

Lemma 4. The subgroup $L=\left\langle a, a^{v}\right\rangle$ is isomorphic to the group $L_{2}\left(p^{n}\right)$ for some $n$. 
Proof. It is clear that $|K: L| \leqslant 2$. According to Lemma $1, P=L \cap U$ and $P_{2}=L \cap U^{x}-$ elementary Abelian Sylow $p$-subgroups of $L$, with Silov $p$-subgroups of $L$ are pairwise coprime, in particular, $L$ is not an abelian group.

It is clear that $B_{1}=N_{L}(P)=L \cap B$. If $B_{1}=P$, then $P \cap P^{x}=1$ for any $x \in L \backslash P$, and by the Frobenius theorem $L=M \lambda P$ is the Frobenius group with nilpotent kernel $M$ [15, Thompson's Theorem 1.5] and the cyclic complement $P=\langle a\rangle$ [15, Burnside's Theorem 1.2]. By Lemma 2, the 2-rank of the group $K$ (and the group $L$ ) does not exceed 2, and if $2 \in \pi(M)$, then the order of the center of a Sylow 2-subgroup from the Frobenius kernel $M$ is 4 . By the conditions $p>3$ and, therefore, $2 \notin \pi(M)$.

Obviously, $|B \cap K|=2 p$ and by Frattini's argument and Lemma $1 N_{K}(P)=\langle a\rangle \lambda\langle k\rangle=D-$ dihedral group, where $k \in v^{K}$. Hence, by virtue of the same Burnside theorem [15, Theorem 1.2] $C_{Z}(k) \neq 1$ for the center $Z$ of each Sylow $q$-subgroups of $M$. Obviously, $C_{Z}(k)<H^{x}$ for some $x$, and in view of item 4 of Lemma $1,\left|\Omega_{1}(Z)\right|=q$. Hence, the dihedral group $B \cap P$ is contained in the group of automorphisms of a cyclic group of order $q$, a contradiction, therefore, $B \cap P \neq P$.

Note that by Frattini's argument and Lemma 1 the group $K$ contains the group anyway dihedral $D=\langle a\rangle \lambda\langle k\rangle$, where $k \in v^{K}$. Let $M$ be the minimal normal subgroup in $K$ from $L$. Consider the case when $M$ - elementary abelian $q$-group. As proved above, $q \neq 2$. Since $P$ is strongly isolated in $L=\left\langle P, P^{v}\right\rangle$ as above, we have $q \neq p, M \lambda P$ is a Frobenius group, $P=\langle a\rangle$, $C_{M}(k) \neq 1,|M|=q$ and $D \leqslant$ Aut $M$, a contradiction. Hence, $M$ is a direct product of nonabelian simple groups, and since the 2-rank of the group $M$ does not exceed 2, then $M$ is a simple group of 2-rank 2 .

If $P \notin M$, then by Frattini's lemma $P \cap N_{L}(S) \neq 1$ for some Sylow 2-subgroup $S$ of $M$ and each element from $P^{\#} \cap N_{L}(S)$ acts on $S$ regularly, which is impossible, since the 2-rank of $G$ is at most 2 and $p>3$. Therefore, $P \leqslant M$ and $|L: M| \leqslant 2$, and therefore $M=\left\langle P, P^{v}\right\rangle=L$.

If a Sylow 2-subgroup $S$ in $L$ is dihedral (Lemma 2), then by the Gorenstein-Walter theorem $\left[17\right.$, p. 27] $L \simeq L_{2}(q), q$ is odd, or $L \simeq A_{7}$.

Let's exclude the group $L \simeq A_{7}$. For $p=7$, by Kerby's theorem, $H$ contains a unique subgroup of order 3 , and in $A_{7}$ is an elementary abelian subgroup $E_{9}$, which contradicts Lemma 1. Hence, $p=5$. The involution $k$ inverting a cyclic subgroup of order 5 is obviously contained in $J_{2}$. It is easy to check (see, for example, cite [Proposition 14] LSS), that $C_{L}(k)$ contains the only subgroup $\langle b\rangle \leqslant E_{9}$ of order 3 , which is contained in $H_{k}$. But $E_{9} \leqslant C_{L}(b) \nless H_{k}$, which contradicts Lemma 1 . Therefore, $L$ cannot be isomorphic to $A_{7}$.

Let $L \simeq L_{2}(q)$. If $q \neq p^{n}$ then $P=\langle a\rangle$ and $p$ divides either $q-1$ or $q+1$. Since $C_{G}(P)-$ $2^{\prime}$ is a group, then either $q-1=2 p$ or $q+1=2 p$. Note that then $t \in L \cap J_{2}, C_{L}(t) \leqslant N_{L}(P)$, in this case either $\left|C_{L}(t)\right|=q+1$, or $\left|C_{L}(t)\right|=q-1$. However, this is not possible. Therefore, $L \simeq L_{2}\left(p^{n}\right)$. If $v \notin L$, using Lemmas 1-3 and information from [19, p. 8-10], apparently it can be shown that $K \simeq P G L_{2}\left(p^{n}\right)$.

Let a Sylow 2-subgroup $S$ in $L$ be not dihedral. Since $v \in J_{2}$, in view of item 6 of Lemma 2, this means that $J \cap L \subset J_{2}$. As Alperin, Brower and Gorenstein proved [20] finite simple groups of 2-rank 2, up to isomorphism, are the following groups: $L_{2}(q), A_{7}, L_{3}(s), U_{3}(r), M_{11}, U_{3}(4)$, where $q, s, r$ are odd and $q>3$.

First, let's exclude the groups $U_{3}(4)$ and $M_{11}$ from this list. In $U_{3}(4)$ all involutions are conjugate and the Sylow 2-subgroup $S$ is of order 64, all its involutions lie in the center of $Z$, $|Z|=4$ (see, for example, [18, Proposition 13]). If $v \in L$, then $Z^{\#} \subset J_{2}$, which contradicts Lemmas 1, 2. If $Z^{\#} \subset J_{0}$, then $v \notin L$, which contradicts Lemma 2. In $M_{11}$ all involutions are conjugate, the Sylow 2-subgroup $S$ is a semidihedral group of order 16 and the centralizer of the involution is isomorphic to $G L_{2}(3)$ (see, for example [18, clause 14]). As noted above, $J \cap S \subset J_{2}$. Therefore, $S<N_{k}$, where $k$ is the central involution from $S$.

The group $S$ contains a cyclic subgroup of index 2, suitable for the role intersection of $S \cap H_{k}$, but each involution from $S \cap H$ centralizes an element of order 4 in $S \cap H_{k}$, which is impossible by Lemmas 1, 2. Hence, $L$ cannot be isomorphic group $M_{11}$. 
Assume that $L$ is isomorphic to $L_{3}(s)$, or $U_{3}(r)$. Then, by [18, Proposition 11], all involutions and quadruple groups in $L$ are conjugate, $L$ contains an element of order 8 and a Sylow 2-subgroup $S$ in $L$ is isomorphic to either a semidihedral group

$$
\begin{gathered}
S D_{m}=\left\langle s, k \mid s^{2^{m+1}}=k^{2}=1, s^{k}=s^{-1+2^{m}}\right\rangle, m \geqslant 2 \text {, or woven group } \\
W R_{m}=\left\langle s_{1}, s_{2}, k \mid s_{1}^{2^{m}}=s_{2}^{2^{m}}=k^{2}=1, s_{1} s_{2}=s_{2} s_{1}, s_{1}^{k}=s_{2}, s_{2}^{k}=s_{1}\right\rangle, m \geqslant 3 .
\end{gathered}
$$

Recall that in the case under consideration $S \cap J \subset J_{2}$ and, therefore, $S \leqslant N_{j}$ for the involution $j \in Z(S)$. In the group $S=W R_{m}$ from (2), each subgroup of index 2 contains the subgroup $E_{4}$, which is impossible by Lemma 1. And in the cyclic subgroup of order 8 from the group $S=S D_{m}$ is a subgroup of order 4 commuting with all involutions from $S$, which again contradicts Lemma 1. Therefore, in all cases $L \simeq L_{2}(q)$. As proved above, $q=p^{n}$, and the lemma is proved.

Lemma 5. For any element $c \in U^{v}$ the subgroup $L=\langle a, c\rangle$ is isomorphic to the group $L_{2}\left(p^{n}\right)$ for some $n=n(a, c)$.

Proof. By virtue of the finiteness condition for the element $a$ and items 1-2 of Lemma 1 the subgroup $L$ is finite. Further, as in the proof of Lemma 4, $P=L \cap U$ and $P_{2}=L \cap U^{x}-$ elementary Abelian Sylow $p$-subgroups in $L$, Sylow $p$-subgroups in $L$ are pairwise coprime and $L$ is not an abelian group. To continue to follow the logic of the proof of Lemma 4 , we prove that the 2-rank of the group $L$ does not exceed 2. If $L \cap J_{2}$ is nonempty, then the desired follows from Lemma 2. Let $L \cap J_{2}=\emptyset$. Note that by claim 3 of Lemma 1 the involution $z \in H$, and by claim 1 of the same lemma, $z$ inverts the elements $a$ and $c: a^{z}=a^{-1}, c^{z}=c^{-1}$. Therefore, $z \in N_{G}(L)$, the subgroup $K=\langle a, c, z\rangle$ is finite, $|K: L| \leqslant 2, K \cap J_{2} \neq 2$ and for $K$ the boundedness of the 2-rank follows from Lemma 2. Hence, the 2-rank of the group $L$ does not exceed 2, and $D=\langle a, z\rangle$ - dihedral group, $D \leqslant K$. Moreover, in the case $L \cap J=\emptyset$, by Lemma 2 the Sylow 2-subgroups in $K$ (and in $L$ ) are dihedral. Taking into account these remarks, part of the proof of Lemma 4 , on the structure of $L$ groups with dihedral Sylow 2-subgroup, carries over literally to the case under consideration. The lemma is proved.

Lemma 6. For any non-permutable elements $x, s \in a^{G}$ the subgroup $L=\langle s, x\rangle$ is finite and isomorphic to the group $L_{2}\left(p^{n}\right)$ for suitable $n=n(s, x)$.

Proof. Due to the arbitrary initial choice of the element $a$ from the class of conjugate elements of $a^{G}$ it follows that statement of Lemma 5 is true for any $s \in U^{\#}$ and $x \in U^{v} \cap a^{G}=U^{v \#}$. Since $G$ is 3-transitive on the set $U^{G}$, we conclude that that the lemma is true.

Proof of the theorem. According to [19, p. 9] the group $L=\left\langle a, a^{v}\right\rangle$, isomorphic $L_{2}(q)$ by Lemma 4, has $\frac{q(q+1)}{2}$ cyclic subgroups of order $\frac{(q-1)}{2}$ (Cartan subgroups), of these, $(B \cap L) \cup\left(B^{v} \cap L\right)$ contains $2 q-1$ such subgroups. Since $\frac{q(q+1)}{2}>2 q-1$ for $q>3$, then there is a pair of dots $\gamma, \delta \in X \backslash\{\alpha, \beta\}$ for which the intersection $L \cap G_{\alpha \beta}$ is cyclic subgroup conjugate to the Cartan subgroup $L \cap H$ of order $\frac{(q-1)}{2}$. The group $G$ acts on the set $J_{2}$ twice transitively, since it is twice transitive on the set $H^{G}$, and each the subgroup $H^{g}$ is defined by its unique central involution $z^{g}$ from $J_{2}$ (Lemma 1). Hence we deduce that any pair of involutions from $H \cap J_{2}$ is contained in an appropriate subgroup conjugate to the subgroup $L$. This means that the involution $v$ is finite in the group $N$, and by [16, Corollary 2.30] the subgroup $N$ is locally finite. By Theorem 2 in [21], the group $G$ is locally finite. The theorem is proved.

This work was financially supported by the Russian Foundation basic research (grant 19-0100566-a). 


\section{References}

[1] H.Wähling, Theorie der Fastkörper, Essen: Thalen Ferlag, 1987.

[2] M.Hall, Group Theory, Moscow, IL, 1962 (in Russian).

[3] O.H.Kegel, Zur Structur lokal endlicher Zassenhausgruppen, Arch. Math., 18(1967), $337-348$.

[4] V.D.Mazurov, On infinite groups with abelian centralizers of involutions, Algebra and Logic, 39(2000), no. 1, 74-86 (in Russian).

[5] T.Grundhöfer, E.Jabara, Fixed-point-free 2-finite automorphism groups, Arch. Math., 97(2011), 219-223.

[6] A.I.Sozutov, On Shunkov Groups Acting Freely on Abelian Groups, Sib. math. zh., 54(2013), no. 1, 188-198 (in Russian).

[7] A.I.Sozutov, E.B.Durakov, On the local finiteness of periodic exactly triply transitive groups, Algebra and Logic, 54(2015), no. 1, 70-84.

[8] E.Rips, Y.Segev, K.Tent, A sharply 2-transitive group without a non-trivial abelian normal subgroup, 2014, 1-17.

[9] K.Tent, M.Ziegler, Sharply 2-transitive groups, Advances in Geometry, 16(2014), no. 1, 1-5. DOI: 10.1515/advgeom-2015-0047

[10] K.Tent, Sharply 3-tranzitive groups, Advances in Mathematics, 286(2016), 722-728.

[11] A.I.Sozutov, E.B.Durakov, O.V.Kravtsova, On some sharply triply transitive groups, Algebra, logic and applications, Abstracts report Int. Conf., Krasnoyarsk, July 19-25, 2010, 86-89 (in Russian).

[12] E.B.Durakov, Exactly multiply transitive groups, INFORMATION TECHNOLOGIES IN MATH AND MATHEMATICAL EDUCATION. Materials of the IX All-Russian scientific and methodological conference with international participation, Krasnoyarsk State Pedagogical University named after V. P. Astafieva, 2020, 17-21 (in Russian).

[13] A.I.Sozutov, O.V.Kravtsova, On $K T$-fields and exactly triply transitive groups, Algebra and Logic, 57(2018), no. 2, 232-242.

[14] E.B.Durakov, E.V.Bugaeva, I.V.Sheveleva, On Sharply Doubly-Transitive Groups, J. Sib. Feder. University. Mathematics and physics, 6(2013), 28-32.

[15] A.M.Popov, A.I.Sozutov, V.P.Shunkov, Groups with Frobenius systems subgroups, Krasnoyarsk, KSTU Publishing House, 2004 (in Russian).

[16] A.I.Sozutov, N.M.Suchkov, N.G.Suchkova, Infinite groups with involutions, Krasnoyarsk, Siberian Federal University, 2011 (in Russian).

[17] D.Gorenstein, Finite Simple Groups. An introduction to their classification, N. Y., Plenum Publishing Corp., 1982; Translation from English, Moscow, Mir, 1985 (in Russian).

[18] D.V.Lytkina, A.I.Sozutov, A.A.Shlepkin, Periodic Groups of the 2-rank two saturated with finite simple groups, Sibirsk. electron. mat. izv., 15(2018), 786-796 (in Russian).

DOI: $10.17377 /$ semi.2018.15.064 
[19] V.M.Busarkin, Yu.M.Gorchakov, Finite split groups, Moscow, Nauka, 1968 (in Russian).

[20] J.L.Alperin, R.Brauer, D.Gorenstein, Finite simple groups of 2-rang two. Collection of articles dedicated to the memori of Abraham Adrian Albert, Scripta Math., 29(1973), no. 3-4, $191-214$

[21] A.I.Sozutov, E.B.Durakov, Sharply doubly transitive groups with generalized finite elements, Siberian Math. J., 58(2017), no. 5, 887-890. DOI: 10.17377/smzh.2017.58.515

\section{Точно трижды транзитивные группы с конечным Элементом}

Евгений Б. Дураков

Сибирский федеральный университет Красноярск, Российская Федерация

Аннотация. В настоящей работе исследуются точно трижды транзитивные группы. Доказана локальная конечность точно трижды транзитивных групп подстановок характеристики $p>3$, содержащих конечный элемент порядка $p$.

Ключевые слова: группа, точно k-транзитивная группа, точно трижды транзитивная группа, локально конечная группа, почти-область, почти-поле. 